\title{
El Aula Virtual como Estrategia Didáctica en un Mundo Transformado por el Covid-19
}

Jesús Arellano Landeros, Méxicano

jarellanolandereos@gmail.com

https://orcid.org/0000-0002-8740-968X

Universidad Aliat: Naucalpan

Recibido: Olde enero del 2021

Aceptado: 06 de febrero del 2021

\section{Resumen}

El presente artículo describe una propuesta constructiva acerca del nuevo rumbo que están tomando las Instituciones de Educación Superior (IES) para abatir el efecto del confinamiento en nuestro país producido por la pandemia a nivel mundial por COVID-19, donde el principal propósito es la búsqueda de reestructurar y transformar los procesos de enseñanza aprendizaje en la Escuela Normal Superior del Valle de México (ENSVM) sede Nezahualcóyotl para poder continuar laborando en la impartición de catedra, ante el riesgo de la salud de los estudiantes, personal docente y administrativo por medio del uso del aula virtual como estratégica didáctica de comunicación asertiva. Sabemos que las consecuencias que ha traído este fenómeno en torno a la educación son gravemente desfavorables, por lo tanto, consideramos como herramienta inclusiva del uso de las Tecnologías de la Información y Comunicación (TIC) de donde se retoma el uso del aula virtual como la solución más viable, en primer momento, para preservar la salud y favorecer la formación continua de los estudiantes también implementar estrategias para la interacción entre docentes y alumnos, favoreciendo la entrega, recepción, revisión y retroalimentación de tareas. Se pretende continuar trabajando con responsabilidad, siendo inclusivos y empáticos en todo momento, además como principales actores educativos se tiene que hacer frente a este nuevo reto de transformar la manera de impartir clases a distancia, con 
el propósito de abatir el efecto del COVID- $19^{1}$ de manera eficaz, sustituyendo la modalidad presencial con el nuevo modelo de educación a distancia como estrategia y resultado de una investigación descriptiva y cuantitativa la cual fundamenta teóricamente la habilidad docente y el proceso seleccionado para solucionar las dificultades de manera inmediata en caso de estudio en la ENSVM sede Netzahualcóyotl, donde se implementó el uso de la plataforma virtual.

Palabras clave: Aula virtual, estrategia didáctica, transformación, educación superior, COVID19.

\section{Abstract:}

This article describes a constructive proposal about the new direction that Higher Education Institutions (HEIs) are taking to reduce the effect of confinement in our country produced by the global pandemic by COVID-19, where the main purpose is to search of restructuring and transforming the teaching-learning processes at the Escuela Normal Superior del Valle de México (ENSVM), Nezahualcóyotl headquarters in order to continue working in the teaching of professorships, given the risk of the health of students, teaching and administrative use of the virtual classroom as a didactic strategy for assertive communication. We know that the consequences that this phenomenon has brought around education are seriously unfavorable, therefore, we consider the use of Information and Communication Technologies (ICT) as an inclusive tool, from which the use of the virtual classroom as the The most viable solution, in the first place, to preserve health and promote the continuous training of students, also implement strategies for interaction between teachers and students, favoring the delivery, reception, review and feedback of tasks. It is intended to continue working with responsibility, being inclusive and empathetic at all times, in addition, as the main educational actors, we have

\footnotetext{
2 Virus que causa una enfermedad respiratoria llamada enfermedad por coronavirus de 2019 (COVID19). EI SARS-CoV-2 es un virus de la gran familia de los coronavirus, un tipo de virus que infecta a seres humanos y algunos animales. 
to face this new challenge of transforming the way of teaching distance classes, in order to reduce the effect of COVID- $19^{2}$ in an effective way, replacing the face-to-face modality with the new distance education model as a strategy and result of a descriptive and quantitative research which theoretically bases the teaching ability and the selected process to solve the difficulties immediately in case of study in the ENSVM headquarters Netzahualcóyotl, where the use of the virtual platform was implemented.

Keywords: Virtual classroom, didactic strategy, transformation, higher education, COVID-19.

\section{Introducción}

El presente trabajo de investigación muestra cómo se implementa de manera emergente el aula virtual en la Escuela Normal Superior del Valle de México (ENSVM) sede Nezahualcóyotl, con la finalidad de fortalecer la educación continua en los alumnos de las licenciaturas en Educación, Preescolar, Primaria, y Educación Secundaria. La docencia como profesión ha sido abordada por diversas investigaciones y perspectivas (Tenti Fanfani, 2006, 2008; Tardif, 2012; Davini, 2005), entre estas la perspectiva teórica que asume Contreras Domingo (2001, 2010), como ya sabemos México cuenta con una población de más de 120 millones de habitantes siendo uno de los diez países más poblados del mundo, y el tercero en América latina.

Bajo esa perspectiva de población y con base a alcanzar las metas de los programas de Educación Superior mencionadas en la agenda de La Organización de las Naciones Unidas para la Educación, la Ciencia y la Cultura, (UNESCO, 2030) para el Desarrollo Sostenible contemplados en 17 Objetivos, donde se marca la pauta a seguir, para trabajar con las nuevas generaciones del siglo XXI, donde se contempla la implementación de las TIC, en instituciones de nivel superior.

El objetivo principal es formar profesionistas en el área del conocimiento pedagógico educativo cuya duración es en general de cuatro años, los cuales se imparten en esta (ENSVM), sede Nezahualcóyotl de formación para maestros en este caso como Instituciones de Educación 
Superior (IES), en el marco de la investigación descriptiva y cualitativa apoyados en la técnica de análisis de contenidos de documentos contemplando hasta nivel de postgrado que es la última fase de la educación formal y tiene como antecedente obligatorio la licenciatura, que comprende los estudios de especialidad, maestría y doctorado. Se refiere a una formación enfocada en el desarrollo del conocimiento, la innovación y la investigación científica y tecnológica, vinculados directamente con el ejercicio del Servicio Profesional Docente (SPD), estamos trabajando en un enfoque basado en competencias, que nos permite visualizar una mirada de la implementación del aula virtual y de la situación actual en la Escuela Normal Superior del Valle de México (ENSVM), Sede Nezahualcóyotl.

\section{Materiales y métodos}

Para llevar a cabo esta propuesta de innovación e intervención buscamos la metodología que nos ayude a favorecer el aprendizaje en el nuevo contexto educativo a distancia, donde se abordan algunos aspectos que considero relevantes mencionar como son:

a) Los contextos internos y externos de nuestra escuela en cuanto a la actuación de sociedades del conocimiento contemplando la importancia del aula virtual y su uso como agente transformador en la educación superior considerando que es un recurso que posibilita altos niveles de interactividad entre docentes y alumnos, así como la comunicación en todo momento para lograr un mejor aprovechamiento de los recursos tecnológicos hay dos tipos de aprendizaje en esta modalidad siendo el aprendizaje sincrónico cuando se utilizan las videoconferencias realizando actividades grupales y debates por lo general, el aprendizaje asincrónico es más autodirigido y se da en plataformas donde los contenidos y las instrucciones ya están predefinidos para el alumno y es el que decide a qué hora aprender.

b) El aula virtual pasó de ser un complemento de apoyo en las aulas presenciales llegando a convertirse en la parte fundamental que daría solución a la problemática que se vivía en esos momentos, siendo de vital importancia su uso y manejo en las actividades 
educativas quedando como alternativa para solventar esta crisis del confinamiento, destacando así la importancia del uso y adecuación al currículo por los grandes beneficios y oportunidades de comunicación que otorga a la comunidad estudiantil.

c) Las clases en línea fueron impulsadas por medio del uso del aula virtual, retomando las actividades que se venían trabajando desde el inicio del año escolar 2020 transformando así la forma de trabajo de docentes y alumnos, ahora su medio de interacción será el uso del internet a través de dispositivos electrónicos. Desde ese momento la capacitación de alumnos y docentes relacionada al uso de estos ambientes virtuales de aprendizaje tiene la finalidad de datarlos de las habilidades digitales para desempeñarse en la nueva modalidad.

d) Lo que comenzó como un proyecto de innovación de apoyo en las prácticas virtuales en la comunidad de la ENSVM sede Nezahualcóyotl, pasó a transformar la realidad educativa en un periodo muy corto de tiempo. Por esta razón se llevó acabo esta implementación del aula virtual como estrategia didáctica en un mundo transformado por el COVID-19. Ante este acontecimiento de magnitud global aprendimos a identificar y aplicar actividades lúdicas que nos ayudan a mejorar y contribuyan al desarrollo de competencias empleando las Tecnologías de la Información y Comunicación (TIC).

Una vez que se vio la necesidad de cambiar el paradigma educativo presencial al paradigma educativo virtual en el cual se brindaron las herramientas digitales a docentes y alumnos para migrar de manera emergente a este nuevo contexto, ya no como un apoyo si no como la nueva herramienta para mantener la comunicación, interactiva y lograr tener un desempeño eficaz en el aula virtual, es en ese tenor que acudimos a efectuar nuestro estudio de factibilidad o dicho de otra forma la realización del diagnóstico "proceso a través del cual conocemos el estado o situación en que se encuentra algo o alguien, con la finalidad de intervenir, si es necesario para aproximarlo a lo ideal. Resulta un punto de apoyo insustituible para iniciar la acción (...) ya 
que revela las condiciones y apunta las direcciones en que se debe desarrollar el proceso" (Luchetti, 1998, pág. 17).

Para dar paso a una respuesta efectiva, se desarrolla como primer elemento esta intervención en nuestra investigación, considerando las recomendaciones de la agenda 2030 de La (UNESCO), tomando como referencia que a pesar de que los profesores son la columna vertebral del sistema educativo nacional, esta profesión resulta cada vez menos atractiva para los estudiantes jóvenes, lo que provoca un envejecimiento del conjunto del profesorado, esta postura la podemos ver en un estudio sobre todo en los niveles educativos más avanzados, por ejemplo la media en los países de la OCDE, es un $33 \%$ de los profesores de educación primaria a secundaria tenía al menos 50 años en el año 2015, lo que representa un incremento de 3 puntos porcentuales con respecto a 2005 .

En el contexto actual es imprescindible para la ENSVM abrir más la matricula estudiantil por los efectos del COVID-19 que cada día termina con la vida de más ciudadanos, siendo que un cierto porcentaje se dediquen al magisterio pudiéndose ver claro en las estadísticas de decesos a nivel nacional, aunque no se aborda con profundidad, es importante mencionar que de alguna manera esto está ligado a la transformación educativa, ya que debido a los altos índices de decesos de docentes algunos adultos quienes además de tener dificultades con el uso y manejo de las (TIC), aplican un concepto y una práctica educativa distinta a la de los nuevos docentes que se formaron en la era digital en la que el mundo se está desarrollando y que traen consigo nuevas visiones, ideas innovadoras y propuestas que pueden contribuir al desarrollo de esta nueva modalidad educativa, cabe resaltar que este fenómeno y la deserción de alumnos de educación superior en México va en aumento por la crisis que inicio en el año 2020, es fundamental darle continuidad al sistema de aprendizaje a distancia inclusivo y efectivo como se ha venido manejando sin embargo sabemos no hay nada que reemplace la experiencia escolar en persona por ese motivo tenemos la necesidad de motivar a los alumnos de forma productiva y fructífera para mejorar sus aprendizajes adquiridos en esta nueva modalidad de enseñanza virtual. 
En ese contexto sumando estos hechos, que tienen relación directa con el quehacer docente, no podemos dejar de insistir en continuar trabajando en la formación integral de profesionales de la educación, siendo la ENSVM sede Nezahualcóyotl una de las principales instituciones formadoras de los nuevos docentes que se incorporaran a la labor magisterial incentivando de conocimientos a las comunidades de nuestro país. Por otro lado hacer el llamado a redoblar esfuerzos para que se logre incrementar la selección de nuevos candidatos a estas licenciaturas de formadores de docentes de nivel básico, innovando y creando ambientes de aprendizaje virtuales cada vez mejor estructurados y apegados a los nuevas sociedades del conocimiento conforme al modelo pedagógico emergente del cual estamos haciendo uso con mayor interactividad entre asesores de asignatura y alumnos.

En el contexto de las (IES) del siglo XXI, contemplamos el uso de las (TIC), y sus nuevas aplicaciones en el entorno educativo donde aparece el concepto de Tecnologías del Aprendizaje y del Conocimiento (TAC), y las Tecnologías para el Empoderamiento y la Participación (TEP) utilizadas para fortalecer la educación continua en la comunidad escolar en México donde se viven diversos contextos educativos como consecuencia del confinamiento en todo el territorio nacional, los docentes viven de manera aislada los problemas y carencias que se enfrentan en las aulas provistas en su domicilio mediadas por la tecnología, con el compromiso de hacer esta labor innovadora y continuar con la impartición de conocimientos dirigidos a los estudiantes como solución emergente ante esta situación de confinamiento que incluye a todas las regiones y escuelas que integran nuestro país, siendo los principales afectados los estudiantes de las diferentes licenciaturas que cursan su semestre de manera presencial y que fueron orillados a migrar a las clases virtuales. Es importante mencionar que en sus hogares algunos no cuentan con instalaciones (habitación propia) y medios de comunicación e Internet para llevar a cabo su trabajo en línea.

Es en este tenor que hablaremos de la ENSVM Sede Nezahualcóyotl donde se llevó a cabo la implementación del aula virtual. Realizando como primera actividad un diagnóstico del contexto, basándose en la metodología de la técnica FODA, expuesta como guía recomendada 
para dar inicio a detectar la las fortalezas y áreas de oportunidad para llevar a cabo esta propuesta mediada por la tecnológica por medio de un gestor de aprendizaje que concluye con el tema de la educación a distancia.

Así mismo nos muestra las posibilidades que tenemos de seguir transformando nuestra labor docente tomando como base la inclusión al modelo a distancia por medio de la plataforma virtual de aprendizaje, la cual nos permite la posibilidad de llevar acabo nuestra labor docente y también incorporarnos a las sociedades del conocimiento por mediado por las TIC, mitigando con ello el efecto por la crisis de salud del COVID-19, por este motivo se realizó la suspensión de todas las actividades grupales, eventos masivos como también las clases presenciales en todo el territorio nacional, es así como se comenzó a dimensionar el alcance que tienen las aulas virtuales como estrategia didáctica en un mundo transformado por el COVID-19, y gracias a esta implementación existe interacción y comunicación en tiempo real con la comunidad estudiantil dando paso firme al propósito de abatir el confinamiento y continuar formando a los alumnos en su carrera profesional incidiendo en los procesos educativos de desarrollo humano y social de la comunidad estudiantil.

\section{Resultados}

El objetivo de esta investigación es describir las experiencias de los estudiantes y docentes vividas en el periodo de marzo a diciembre del año 2020, respecto al uso de las herramientas tecnológicas que facilitaron la comunicación e interacción para dar continuidad a los programas académicos, dando a conocer como se transformaron los escenarios de clases presenciales a estrategias de aprendizaje autodirigidas y autogestivas por medio del aula virtual, la cual se fue transformando empleando prácticas didácticas aplicados a los nuevos modelos pedagógicos a distancia con la firme decisión de seguir impartiendo conocimiento por medio del aula virtual.

El trabajo de campo se llevó a cabo con estudiantes y profesores de las licenciaturas en educación preescolar, primaria y educación secundaria impartidos en la (ENSVM), sede Nezahualcóyotl, en el Estado de México durante el primer y segundo periodo del año 2020. 
Tomando en consideración el entorno educativo donde la UNESCO integró un portal con soluciones para la comunicación y el aprendizaje a distancia (UNESCO, 2019). En su portal (https://en.unesco.org/covid19/ educationresponse/solutions), dan la pauta de sugerencias como alternativas de respuesta a nuestro problema local, de los cuales abordaremos los puntos más importantes por mencionar algunos:

- Sistemas de gestión de aprendizaje digital

- Contenido de aprendizaje autodirigido

- Plataformas de colaboración de video en vivo

- Herramientas para que los maestros creen contenido de aprendizaje digital

Dentro de estos cuatro ejes podemos darnos cuenta que se cubre con este proyecto lo antes señalado, como alternativa de solución el aula virtual es viable y su utilidad para obtener buenos resultados en los procesos educativos resolviendo con esto la principal problemática de la imposibilidad de poder asistir a clases presencialmente por la crisis sanitaria que prevalece en nuestro país, por lo anterior tenemos que mejorar las estructuras de las aulas tradicionales, siendo más flexibles en la inclusión de los entornos virtuales para seguir trabajando replanteando la utilización de las herramientas virtuales como recursos educativos metodológicos para favorecer el aprendizaje y obtener una mejora en los resultados académicos de los alumnos fomentando la inclusión, motivación, empatía y autonomía del aprendizaje en la comunidad en la ENSVM sede Nezahualcóyotl.

Teniendo como antecedente que este fenómeno está afectando familias en la mayoría de los casos a personas mayores, padres o madres de familia, y a maestros de todos los niveles educativos tenemos que ser conscientes de esta situación siendo empáticos, sensitivos y humanistas ya que en el presente semestre hay muchos casos que afectaron a varios alumnos o familiares directos, con esto desmotivando por completo y atenuando sus emociones bajando el interés por continuar en las sesiones de aprendizaje para atender sus necesidades socioemocionales y económicas qua también son de relevancia para el aprovechamiento escolar. 
En cuanto al desempeño docente los temas son de gran importancia y mediados por las tecnologías nos damos la oportunidad de crear conferencias donde acordamos que el desarrollo procesal práctico de un currículo es fundamental para comprender desde su gestión, la constitución y formación flexible del modelo pedagógico de acuerdo a las posibilidades de la tecnología y el marco organizativo en cada a una de las instituciones educativas, concretamente cuando transformamos el modelo de enseñanza presencial a un modelo de enseñanza en línea tenemos que hacer el acuerdo sobre los criterios y formas de evaluación curricular que se van a trabajar los cuales deben ser:

\section{- $\quad$ Ser permanentes}

- $\quad$ Ser de importancia en la mejora educativa

- Trabajar en academias de acuerdo al contexto, (videoconferencias).

- Conocer los retos relacionados al diseño curricular y generar nuevas propuestas para esta nueva modalidad de la cultura digital.

En el contexto se tomaron en cuenta los resultados que arrojaron las encuestas que se muestran la siguiente información de acuerdo a las preguntas del formulario aplicado a estudiantes del nivel superior en las tres licenciaturas que se imparten dentro de la ENSVM sede Nezahualcóyotl, del cual fueron considerados tres de grupos de alumnos, de primer semestre realizando este cuestionario en línea mediante las TIC, a los estudiantes y docentes.

En el cual se busca verificar la eficacia y uso del aula virtual así como verificar la conexión a internet desde su casa, aprovechando el acercamiento con los alumnos para poder establecer las bases que apoyen a la implementación del aula virtual y mejora del uso de habilidades digitales para entrar al nuevo modelo pedagógico emergente de educación a distancia, donde se deben de valorar todos los medios y recursos para reestructurar la forma de enseñanza-aprendizaje, 
por el momento solo se hicieron encuestas de lo más importante que nos afectaba en estos momentos. Véase figuras 1, 2 y 3 graficas del manejo en habilidades digitales Tics.

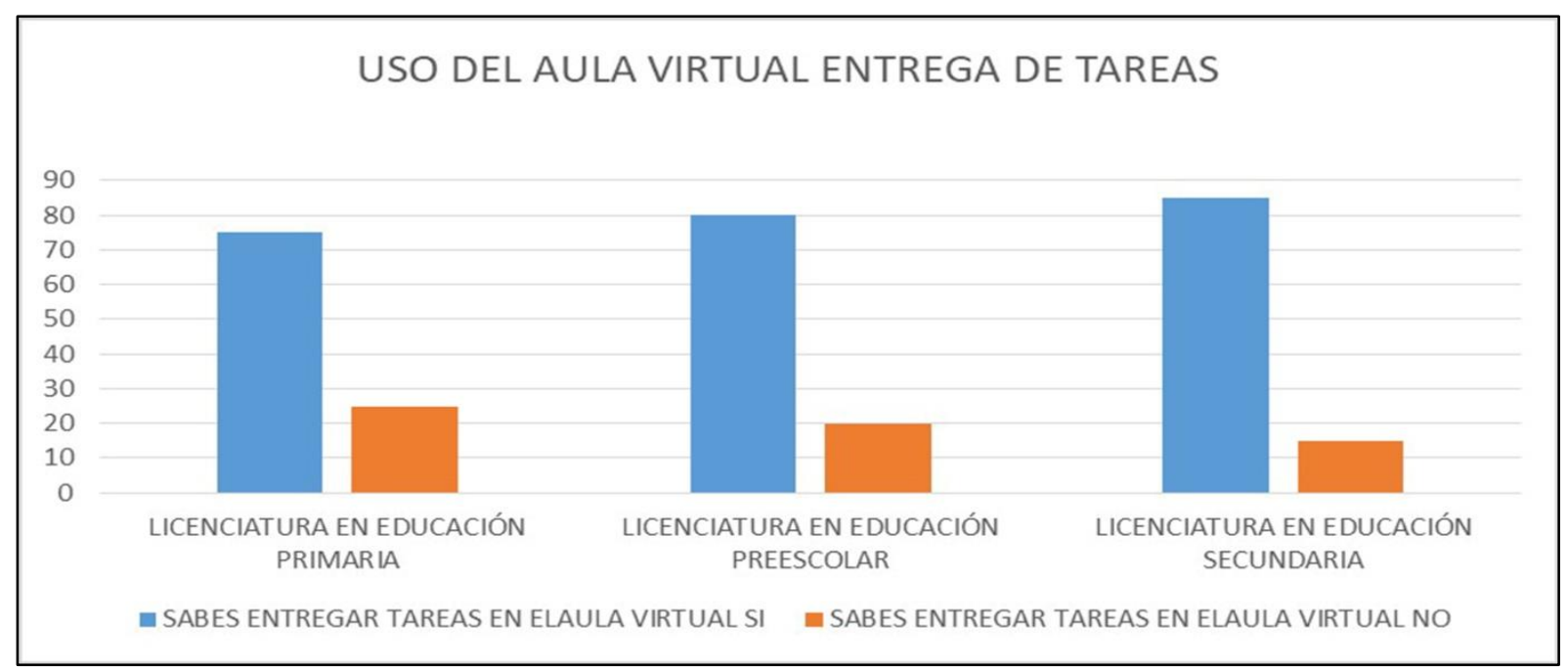

Figura 1. Entrega de tareas en aula virtual. Fuente: Elaboración propia.

Como podemos observar en la gráfica 1 se puede ver que la mayoría de los alumnos tienen la posibilidad de realizar sus entregas de tareas, por otro lado, al momento de ver las respuestas que nos dio la aplicación de la encuesta acerca del uso de la plataforma y la entrega de tareas en línea, se puede observar que si tienen conocimiento y habilidades para realizar sus actividades tanto alumnos como docentes que se incorporan a esta nueva modalidad educativa.

Es por este motivo que se dio a la tarea de investigar el contexto de los estudiantes por medio de las encuestas, la primera intención es saber si la comunidad cuenta con los requerimientos mínimos para llevar acabo la conexión apropiada para brindar servicios de calidad en modalidad virtual, que como ya se abordó anteriormente es el vehículo principal, además es importante mencionar el gran impacto que genera contar con el paquete básico de internet que está en promedio de los quinientos pesos, dependiendo de la compañía que les brinde el servicio, repercutiendo el bolsillo en los hogares de los alumnos y docentes. 
Estos entornos favorecen en la interacción social debido a que tienen un esquema de aprendizaje colaborativo entre estudiantes, docentes y la comunidad (Avello Martínez y Duart, 2016). Véase figura 2. Conexión de internet en la casa de los alumnos.

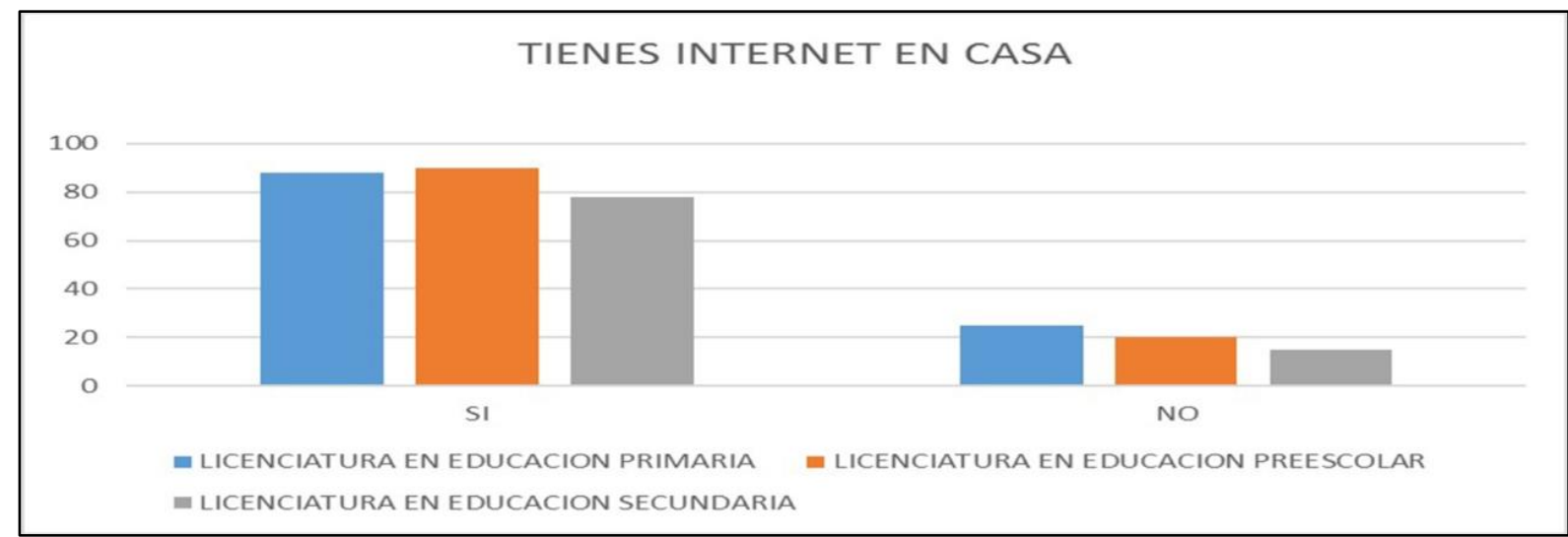

Figura 2. Conexión de internet en casa. Fuente: Elaboración propia.

En las figuras 2 y 3 se muestra la viabilidad de la implementación y el uso de las TIC, donde se contempla que la mayoría de los alumnos pueden establecer comunicación por medio de sus dispositivos a las plataformas virtuales en las cuales adquieren habilidades digitales para llevar a cabo esta labor en esta nueva modalidad educativa, cabe mencionar que también el docente debe de tener los medios necesarios para estar a la par de sus alumnos y así establecer la comunicación ya sea síncrona o asincrónica, de la cual tenemos la evidencia de comunicación con el docente que tiene a su cargo los grupos asignados y por medio de las plataformas pueden interactuar en forma directa con los docentes correspondientes. Véase Figura 3. Aplicaciones de comunicación. 


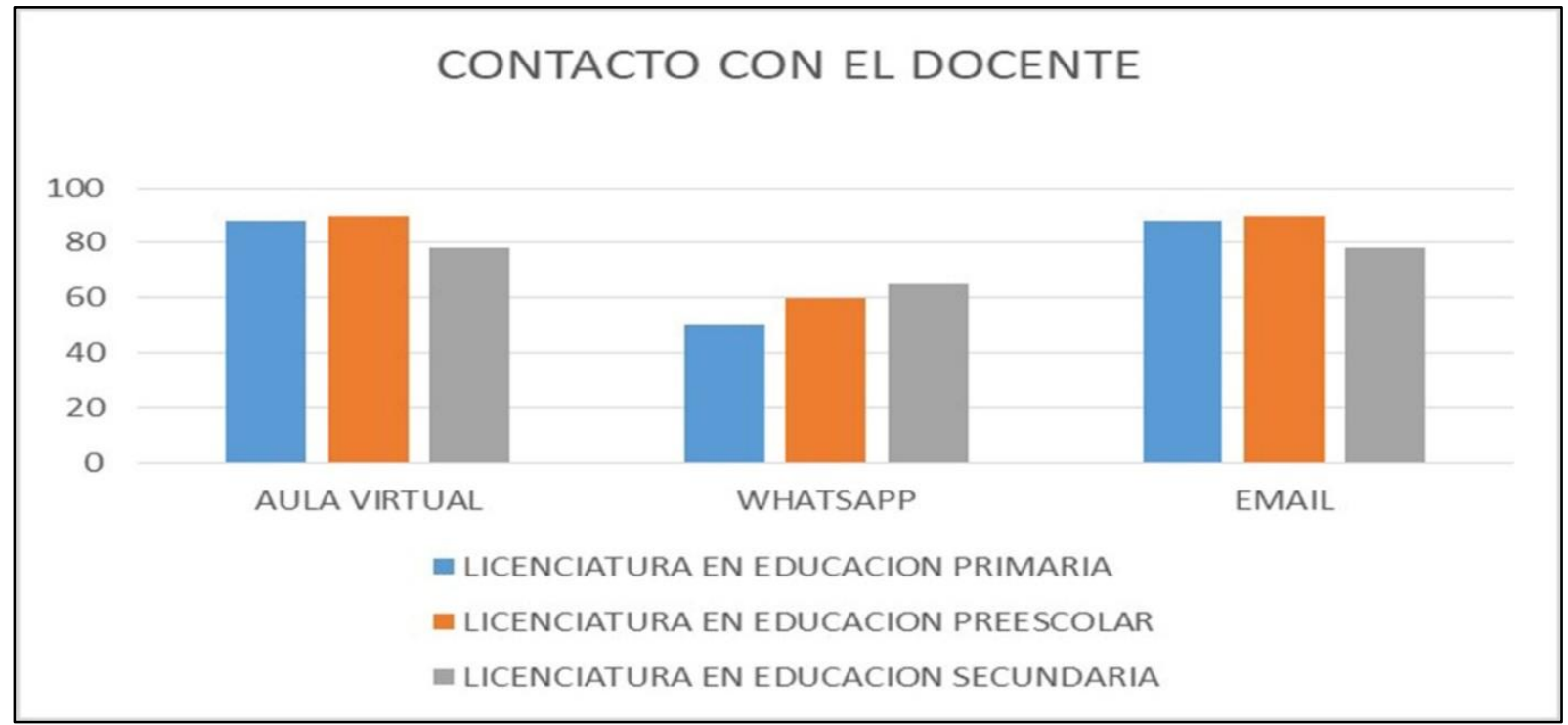

Figura 3. Aplicaciones de comunicación. Fuente: Elaboración propia.

Con base a los instrumentos realizados a los diferentes grupos que constituyen la comunidad escolar y practicas propias de la escuela, se decidió aprobar esta implementación al currículo el proyecto de aula virtual, dirigido a maestros y alumnos, en formación de la ENSVM, Sede Nezahualcóyotl, considerando puntos importantes desarrollados en el diagnóstico, el cual se realizó por medio de la matriz de FODA.

El presente proyecto de aula virtual está estructurado bajo el enfoque basado en competencias y su implementación tiene como benefactores al cuerpo de alumnos y docentes de los diferentes niveles académicos que tiene esta institución de estudios superiores y su modalidad se plasman de la siguiente forma: Véase Tabla 1. Beneficios a la comunidad estudiantil. 
Tabla 1. Beneficios a la comunidad estudiantil. Fuente: Elaboración propia.

\begin{tabular}{|c|c|c|}
\hline CARRERA & MODALIDAD & NUEVA MODALIDAD \\
LICENCIATURA & PRESENCIAL & LÍNEA \\
\hline EDUCACIÓN & & LÍNEA \\
PREESCOLAR & PRESENCIAL & LÍNEA \\
\hline EDUCACIÓN & & \\
\hline PRIMARIA & PRESENCIAL & \\
SECUNDACIÓN & & \\
\hline
\end{tabular}

¿Que se busca favorecer con el aula virtual utilizada en al ENSVM sede Nezahualcóyotl? La inclusión de profesores y alumnos en su uso, ejecución de actividades y adecuación de los planes y programas contemplados en la malla curricular, así como la reestructuración de planeaciones didácticas de cada asignatura y adecuación de las estrategias didácticas al nuevo modelo educativo, por lo tanto, los docentes tienen que evaluar y certificar los conocimientos y actividades realizadas. Una de las metas deseadas de este proyecto que actúa como recurso de aprendizaje y enseñanza, es contemplar los beneficios de estos recursos tecnológicos impartiendo cursos y verificando los aprendizajes adquiridos por los alumnos en la nueva modalidad de enseñanza. Véase figura 4. Componentes de un Centro de Atención Universitario, (C. A U). 
Componentes de un CAAU

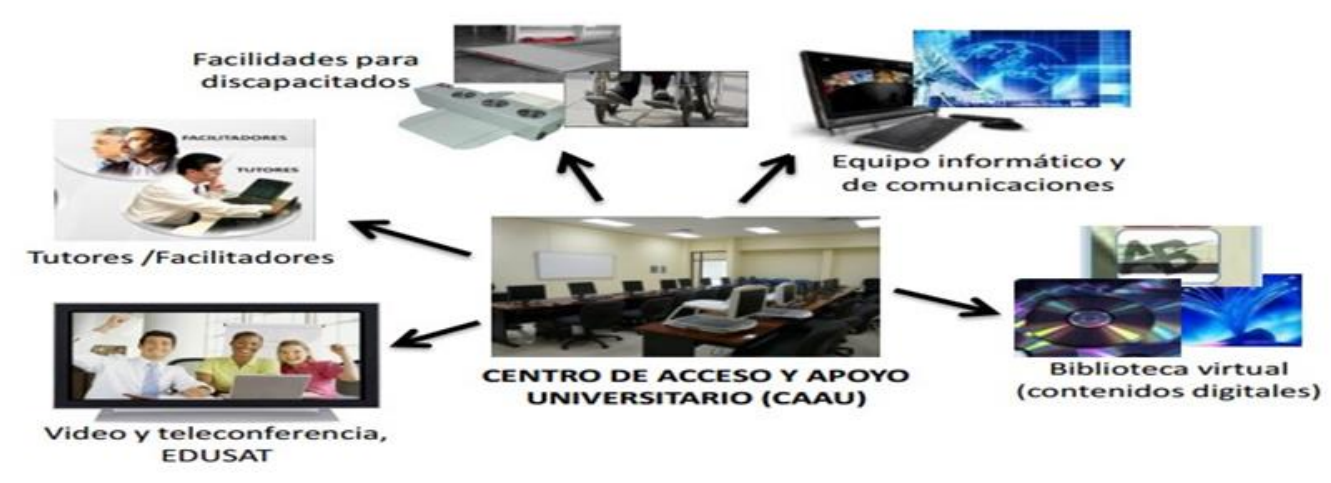

Figura 4. Componentes de un (C.A.U). Fuente: Tomado de http://www.sep.gob.mx/work/mod

En la ENSVM existe la necesidad de trabajar con la plataforma virtual, siendo imprescindible como herramienta principal que brinda a los alumnos el enlace de comunicación para poder continuar impartiendo clases a distancia.

Con base a reforzar su proceso formativo profesional docente, fomentando el uso de las tecnologías tanto en docentes como alumnos y dar mejores servicios de calidad educativos a la comunidad estudiantil, en otro tenor se capacita a los docentes como técnico pedagogos con el fin de que innovar y crear sus propios materiales digitales para sus asignaturas en línea y que puedan aportar para trascender de docentes tradicionales a tutores en línea.

En la figura 5 se puede apreciar la página principal de este proyecto de innovación en beneficio de la comunidad estudiantil y cuerpo docente que desarrolla sus actividades diariamente para la enseñanza y aprendizaje en línea. 


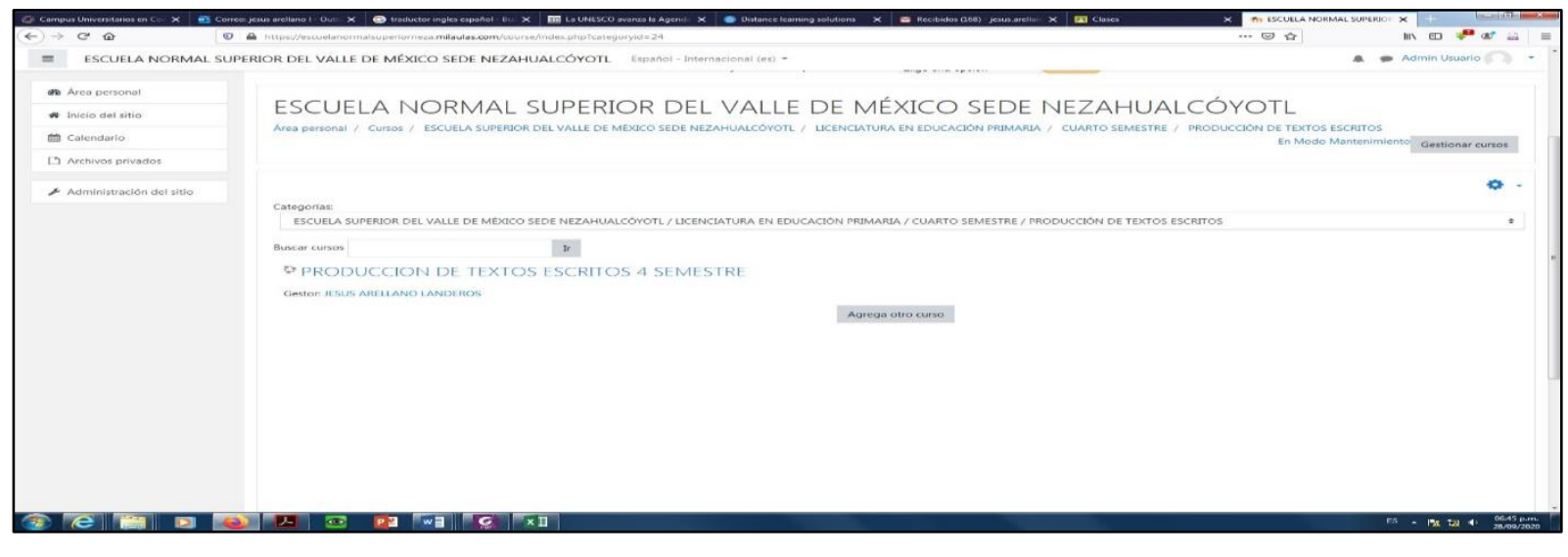

Figura 5. Aula Virtual ENSVM sede Nezahualcóyotl Fuente: Tomado de: https://escuelanormalsuperiorneza.milaulas.com

La figura 5, muestra el sitio del aula virtual donde se está trabajando con los alumnos y docentes con el aula de entrenamiento y capacitación, en un principio y en otras plataformas como Google Classroom para migrar más adelante y cumplir con el convenio hecho por las autoridades educativas en las cuales empezaremos a subir y tener alojados los planes y programas de estudio correspondientes a las asignaturas para que el docente pueda trasformar estos recursos digitales en un aprendizaje significativo que motive a los alumnos y se logren los aprendizajes esperados.

Lo anterior tiene la finalidad de poder llevar a cabo el proceso de transformación, apoyándolos con cursos de actualización y manejo de habilidades digitales para agilizar por medio de la educación a distancia los saberes, haciendo uso de videoconferencias y otras herramientas, logrando resultados positivos por medio de las plataformas virtuales como mediadoras de aprendizaje entre docentes y alumnos.

Por esta razón resulta ser conveniente este proyecto, tomando en cuenta que se busca asignar una mayor precisión al abordaje de los contenidos, brindando la asesoría correspondiente a cada uno de los grupos conformados en el aula virtual de manera permanente como se realizaba en clases presenciales entrando en la transformación de la enseñanza mediada por la tecnología, considerando que es necesario asegurar la mejora de la calidad en la formación profesional de los alumnos. 
Tomando en cuenta la petición de la comunidad escolar de contar con un apoyo y orientación eficaz para los alumnos por medio del personal de asesores pedagógicos quienes recolectan las planeaciones didácticas que hacen los docentes dependiendo de la materia, y así poder dar el proceso de revisión y cotejo de las planeaciones y estrategias didácticas impartidas en el aula virtual, conformando el proceso de evaluación considerando que como docentes tenemos que programar en la planeación actividades que proporcionen un aprendizaje de tipo teórico conceptual y memorístico e interactivo el cual debe ser orientado a una nueva modalidad virtual de acuerdo a los planes y programas vigentes para dar continuidad a todo lo establecido en el curriculom y adecuación del mismo.

Haciendo una reflexión, situamos a los alumnos como el primer elemento de atención, apegándonos a los nuevos modelos educativos basados en competencias. Como ya se ha mencionado muchas veces, la "competencia" es la habilidad para realizar tareas y roles requeridos según los estándares esperados, citando a Roegiers (2010).

La competencia es la posibilidad que tiene una persona de movilizar de manera interiorizada un conjunto integrado de recursos con vistas a resolver, es decir, una familia de situacionesproblema. Esta definición señala la posibilidad como una capacidad que potencialmente se encuentra en la persona enfatiza el aspecto de recursos que se movilizan ante una determinada situación e introduce la idea de familias de situaciones.

Es así como se pretende establecer ambientes de aprendizaje enriquecidos con la tecnología y ofrecer al docente las nuevas formas de enseñanza en las competencias digitales en aulas virtuales con un sentido didáctico pedagógico que le permitan diseñar distintos escenarios de interacción que se verán reflejados en experiencias significativas, diseñando ambientes de aprendizaje por medio de la interacción de diversas formas (alumno-alumno, alumno-profesor, alumno- contenidos).

Por otro lado, Esquivel I. (2013), manifiesta: “...la Educación online ha mostrado mayor efectividad rompiendo las barreras del tiempo y espacio, al ofrecer métodos, técnicas y recursos 
que hacen más efectivo y flexible el proceso enseñanza - aprendizaje, a través del uso de tecnologías como la radio, la televisión, el vídeo, los sistemas de informática y software interactivos..."

Es por esta razón que gracias a la implementación de este proyecto educativo en línea se puede lograr el objetivo fundamental que busca la mejora en la calidad en los servicios educativos ofrecidos favoreciendo el proceso continuo e interrumpido para el bienestar de la comunidad estudiantil de la ENSVM sede Nezahualcóyotl.

\section{Conclusiones}

En la actualidad los docentes y alumnos en formación, no estaban acostumbrados a trabajar en un cien por ciento en línea ni se tenía definido el programa de TIC para darle al docente las herramientas necesarias para afrontar el fuerte reto que conlleva la educación a distancia, en la ENSVM sede Nezahualcóyotl, debido al confinamiento del contexto mundial, se contempló esta medida de impartir clases por medio del aula virtual, quedando en sus inicios como Modelo de Educación Hibrida (MEH), mediado por las TIC, con el asesoramiento y habilidades docentes se realiza el acompañamiento virtual que consiste en crear sesiones de aprendizaje significativas y motivadoras para innovar así la educación tradicional que pasa a ser educación en línea.

Los distintos tipos de educación (curriculum), han intentado responder a las exigencias del proyecto político social y en este caso particularmente los fenómenos o pandemias que afectan a la sociedad, han sido sostenidos en esta situación, principalmente el curriculum de la escuela primaria en México cuyo eje principal es el libro de texto gratuito en la educación básica y en los niveles subsecuentes. En la actualidad, las clases pasan a ser totalmente virtuales en un mundo y comunidades transformadas y amenazadas por la pandemia del COVID-19, de la cual nadie estaba preparado para afrontar estos desafíos educativos, pero se dio esta solución de implementar el aula virtual como estrategia didáctica, que hasta la fecha sigue funcionando como se dio a conocer en el presente trabajo de investigación.

Revista RedCA febrero-mayo 2021

ISSN: 2594-2824 Vol. 3 Núm. 9 


\section{Bibliografía:}

Alemany, D. (2007). Blended Learning: Modelo Virtual-Presencial de Aprendizaje y su Aplicación en Entornos Educativos. España: D. D. Social. Universidad de Alicante. Recuperado de: http://www.dgde.ua.es/congresotic/public_doc/pdf/31972.pdf

Arias, F. (2007). Educación en la globalización: un cambio en la perspectiva. pp. 1-19. Recuperado de: http://www.redalyc.org/pdf/773/77350103.pdf

Avalos, Beatrice, Cavada, Paula, Pardo, Marcela, Sotomayor, Carmen LA PROFESION DOCENTE: TEMAS Y DISCUSIONES EN LA LITERATURA INTERNACIONAL. Estudios Pedagógicos [en linea]. 2010, XXXVI (1), 235-263 Recuperado de: https://www.redalyc.org/articulo.oa?id=173516404013

Avello Martínez, Raidell y Duart, Josep M. Nuevas tendencias de aprendizaje colaborativo en e-learning. Claves para su implementación efectiva. Estudios Pedagógicos. 2016;XLII(1):271-282. Recuperado de: https://www.redalyc.org/articulo.oa?id=1735/173547563017

Castellanos Quintero y Luna Escudero (2009). La internalización y la globalización neoliberal en el contexto de la educación superior en México.Pp. 1-10. Recuperado de: https://ciaed.wordpress.com/2009/06/25/la-internacionalizacion-y-la-globalizacionneoliberal-en-el-contexto-de-la-educacion-superior-en-mexico/

EsquivelI. (2013). Estado del conocimiento sobre la educación mediada por ambientes virtuales de aprendizaje: Una aproximación a través de la producción de tesis de grado y posgrado. Revista Mexicana de Investigación Educativa, Vol.18, No.56, p.249-264

LEYVA, B. (2012) Curso en modalidad de tutorial para la superación del docente tutor de las aulas virtuales. Trabajo de Diploma. UCP Frank País García, Santiago de Cuba. 
Luchetti y Berlanda. (1998). El diagnóstico del aula. Magisterio del Río de la Plata. Buenos Aires.

Mareño Sempertegui, M. y Torrez, V. (2013). Accesibilidad en los entornos virtuales de las instituciones de educación superior universitarias. Virtualidad, Educación y Ciencia, 7 (4), pp. 8-26

Roegiers, X. (2010). Una pedagogía de la integración. Competencias e integración de los conocimientos en la enseñanza. México: Editorial Fondo de Cultura Económica.

UNESCO. (2016). Educación 2030. Declaración de Incheon y Marco de Acción hacia una educación inclusiva y equitativa de calidad y un aprendizaje a lo largo de la vida para todos. $\quad$ http://www.unesco.org/new/fileadmin/MULTIMEDIA/ FIELD/Santiago/pdf/ESP-Marco-de-Accion-E2030-aprobado.pdf

UNESCO. (2019). Education Response to COVID-19 in the Caribbean. Recuperado de: https:// en.unesco.org/covid19/educationresponse/solutions

UNESCO. (2030). La UNESCO Avanza La Agenda 2030 para el Desarrollo Sostenible. Recuperado de: https://en.unesco.org/sustainabledevelopmentgoals 